\title{
Conhecimento do Acadêmico de Medicina sobre Cuidados Paliativos
}

\author{
Knowledge of Medical Students about \\ Palliative Care
}

\author{
Larissa Chaiane Orth ${ }^{1 \odot}$ \\ Eder Yukio Haragushiku ${ }^{\text {I॰ }}$ \\ Inês Carolina Siqueira Freitas ${ }^{1 \odot}$ \\ Micheli Cristiane Hintz ${ }^{10}$ \\ Chaiana Esmeraldino Mendes Marcon ${ }^{I 0}$ \\ José Francisco Teixeira ${ }^{I \oplus}$
}

\section{PALAVRAS-CHAVE}

- Cuidados Paliativos.

- Educação de Graduação em Medicina.

- Internato de Medicina.

- Morte.
Introdução: O envelhecimento da população mundial traz consigo o aumento de doenças crônicas, sem ser acompanhado de melhoria na qualidade de vida ou do enfrentamento das enfermidades. Nesse contexto, os cuidados paliativos se enquadram como um modelo interdisciplinar que visa garantir o cuidado integral ao ser humano. Em contrapartida, a busca incessante pela cura pode resultar em um sentimento de negação e derrota frente à finitude da vida. Durante o processo de graduação médica, é necessário abordar essa temática para que os futuros profissionais se sintam adequadamente preparados e seguros, proporcionando o melhor para seus pacientes. Objetivo: Avaliar o conhecimento sobre cuidados paliativos dos acadêmicos do internato do curso de graduação em Medicina da Universidade do Sul de Santa Catarina (Unisul), campus Tubarão. Método: Realizou-se um estudo transversal por meio de questionário autoaplicável com 39 questões, agrupadas em 12 questões sociodemográficas e 27 questões objetivas sobre cuidados paliativos. A coleta de dados ocorreu de março a junho de 2018. Foram utilizados os testes de qui-quadrado $\left(X^{2}\right)$, exato de Fisher, razão de verossimilhança, Anova e teste de Tukey. O nível de significância estatística adotado foi de 5\%. Resultados: Participaram do estudo 188 acadêmicos, 56,9\% do sexo feminino, com faixa etária prevalente entre 21 e 25 anos. Caso fossem pacientes oncológicos, 3,2\% (6) dos entrevistados prefeririam a decisão de tratamento tomada somente pelo médico, sendo que, destes, 83,3\% (5) eram do sexo masculino ( $p=0,04) ; 49,5 \%$ (93) dos entrevistados se declararam preparados para enfrentar o processo de morte e luto, sendo que, destes, $54,8 \%$ (51) eram do sexo masculino ( $p=0,005) ; 80,3 \%$ (151) dos acadêmicos negaram ter adquirido habilidades para comunicar más notícias, sendo que, destes, $62,9 \%$ (95) eram do sexo feminino $(p<0,001)$. O décimo semestre do curso apresentou a melhor média de acertos em questões relacionadas à farmacologia do tratamento da dor. A maioria dos acadêmicos considerou importante incorporar conteúdos sobre cuidados paliativos ao currículo, porém 68,1\% não tinham interesse em atuar nessa área. Conclusão: O ensino sobre a temática de cuidados paliativos durante a graduação de Medicina da Unisul resultou em um conhecimento adequado dos acadêmicos do internato, porém se observa dificuldade frente ao processo de morte e insegurança na abordagem de comunicações e na atitude médica. É necessário aprimorar o ensino de competências e habilidades na área, enfatizando o cuidado universal centrado no paciente e não somente na cura de doenças, bem como mobilizar esforços a fim de incentivar a autoestima dos acadêmicos. 


\section{KEY-WORDS}

- Palliative Care.

- Education, Medical, Undergraduate.

- Medical Internship.

- Death

\begin{abstract}
Introduction: The aging of the world's population brings with it the increase in chronic diseases, although without a concomitant improvement in quality of life or management of the diseases. In this context, palliative care constitutes an interdisciplinary care model aiming at the integral care of the human being. In contrast, the incessant search for a cure can result in a sense of denial and defeat when facing death. Approaching this subject is necessary during medical school, so that future professionals feel adequately prepared and secure, thus providing the best care for their patients. Purpose: To evaluate the knowledge on palliative care of undergraduate medical students of Universidade do Sul de Santa Catarina (UNISUL), campus Tubarão. Method: A cross-sectional study was carried out through the application of an anonymous, self-administered questionnaire containing 39 questions, divided into 12 sociodemographic questions and 27 objective questions about palliative care. Data collection was carried out from March to June 2018. The Chi-square test (X2), Fisher's exact test, Likelihood ratio, Analysis of Variance (ANOVA) and Tukey's tests were used. The significance level was set at 5\%. Results: A total of 188 medical students, of which 56.9\% were females, at the prevalent age range between 21 and 25 years old participated in the study. In the case of an oncological patient, $3.2 \%$ (6) of the interviewees would prefer that treatment decisions be made only by the physician, of which $83.3 \%$ (5) were males ( $p=0.04) ; 49.5 \%$ (93) of the interviewees declared themselves prepared to face the process of death and grief, of which $54.8 \%$ (51) were males ( $p=0.005) ; 80.3 \%$ (151) of the students denied having acquired skills to communicate bad news, of which $62.9 \%$ (95) were females ( $p$ <0.001). The students at the 10th semester of the Medical course showed a better average of right answers to questions about the pharmacology of pain management. Most students considered important the incorporation of palliative care content in the curriculum, but $68.1 \%$ of them had no interest in working in this area. Conclusion: In conclusion, the teaching of the palliative care subject during UNISUL's medical course resulted in an adequate knowledge of internship students; however, there is some difficulty when facing the process of death and insecurity when approaching the communication and medical attitude. It is necessary to improve the teaching of capabilities and skills in the area, emphasizing universal care focused on the patient and not only on curing diseases, as well as mobilizing efforts to encourage the students' self-esteem.
\end{abstract}

Recebido em 5/6/19

Aceito em 8/6/19

\section{INTRODUÇÃO}

O processo de envelhecimento da população mundial é apontado como uma das maiores conquistas da humanidade, mas também como um dos maiores desafios a serem enfrentados pela sociedade ${ }^{1}$. Essa característica etária traz consigo o aumento das doenças crônicas não transmissíveis, que causam por volta de $72 \%$ das mortes atualmente no Brasil, fato que representa um desafio para os gestores na área da saúde ${ }^{2,3}$.

Contudo, o aumento da expectativa de vida não vem acompanhado de melhora na qualidade da existência ou no processo das enfermidades ${ }^{4}$. Tendo em vista esse retrato complexo da realidade atual, os cuidados paliativos $(C P)$ se enquadram como um modelo promissor de auxílio aos pacientes que vem ganhando espaço no Brasil na última década ${ }^{5}$.
$\mathrm{O}$ verbo paliar tem como significado o ato abrangente de proteger. Derivado do latim pallium, remete ao manto dos cavaleiros da Idade Média, usado para protegê-los das intempéries pelas jornadas que percorriam, cuja filosofia de hospitalidade era caracterizada pela proteção e objetivava alívio para o sofrimento humano ${ }^{6}$. Essa abordagem teve como pioneira a inglesa Cicely Saunders, que dedicou a vida ao alívio do sofrimento humano. A criação do St. Christophers Hospice, em Londres, em 1967, marcou essa trajetória como o primeiro serviço a oferecer cuidado integral ao paciente, reconhecido até a atualidade como referência nessa área ${ }^{6,7}$.

$\mathrm{CP}$ podem ser compreendidos como uma forma de assistência interdisciplinar à saúde que visa garantir o cuidado integral ao ser humano. Essa abordagem objetiva melhorar a 
qualidade de vida das pessoas que convivem com enfermidades severas que ameaçam a vida, bem como de seus familiares, por meio da prevenção e do alívio da dor e de outros sintomas físicos, e do sofrimento psíquico e espiritual, fornecendo suporte aos pacientes concomitantemente a outros tratamentos clínicos, não sendo, portanto, substituto do tratamento curativo, mas, sim, um aliado ${ }^{8,9}$.

No Brasil, esse movimento está em franco desenvolvimento. Em 2009, o Conselho Federal de Medicina incluiu, em seu novo Código de Ética Médica, os CP como princípio fundamental para a prática médica, sendo que em 2011 a Medicina Paliativa passou a ser reconhecida como área de atuação médica ${ }^{10,11}$.

No ano de 2015, a The Economist publicou um relatório que classificou os países segundo um índice de qualidade de morte, contando com diversas variáveis relacionadas à assistência em CP e o processo do morrer, sendo que o Brasil ocupou a $42^{a}$ posição (de um total de 80 países) ${ }^{12}$. Outro obstáculo a ser vencido é a disponibilidade de drogas para o tratamento da dor, um tópico básico para melhorar a qualidade de vida dos doentes, que é inadequada na maior parte do mundo 9 .

A busca incessante pela cura das doenças e o aprimoramento dos procedimentos tecnológicos na área da saúde culminaram com um sentimento de negação da morte, ignorando as ações que visam a uma terminalidade de vida digna. Nessa perspectiva, a finitude da vida é vista como uma derrota pelos profissionais da saúde e não como um processo fisiológico e natural ${ }^{13}$. Durante a graduação, os acadêmicos da área da saúde recebem, majoritariamente, uma capacitação focada na vida, sendo a morte tratada com censura ${ }^{14}$. Recomenda-se integrar as competências básicas de CP a cada ano do currículo da graduação de Medicina, porém cuidando da sobrecarga curricular enfrentada pelos estudantes ${ }^{15}$.

Desse modo, observa-se a grande relevância do estudo desse tema para o campo da saúde. Entretanto, parte dos profissionais da área não está adequadamente preparada e segura para enfrentar situações que utilizem essa abordagem em seu futuro, sentindo-se impotentes e se distanciando do lado humano que dá sentido à profissão. Com base nesse cenário e no fato de haver poucos estudos voltados a essa temática na região, o presente estudo avaliou o conhecimento sobre $\mathrm{CP}$ dos estudantes do internato médico da Universidade do Sul de Santa Catarina. Para isso, verificou-se a percepção do acadêmico sobre o aprendizado em $\mathrm{CP}$, sobre o processo de morte e o morrer, e avaliou-se o conhecimento acerca do tratamento da dor.

\section{MÉTODOS}

$\mathrm{O}$ presente estudo apresentou delineamento transversal. A amostra total foi de 190 acadêmicos do internato médico do curso de Medicina da Universidade do Sul de Santa Catarina (CM-Unisul), com sede no município de Tubarão, devidamente matriculados, que se dispuseram a participar da pesquisa e que estiveram presentes no momento da aplicação dos questionários. Dos 190 acadêmicos, dois foram excluídos do estudo por não estarem presentes em nenhum dos momentos da coleta de dados, sendo realizadas três tentativas de contato, perfazendo 188 participantes.

Foram escolhidos os alunos do quinto e sexto ano, visto que estes tiveram a temática pertinente na disciplina de Sistema Hematopoiético e Bases da Oncologia durante o sétimo semestre, bem como entraram em contato com pacientes internados em âmbito hospitalar, muitos dos quais em tratamento paliativo, podendo colocar em prática o conhecimento adquirido.

A coleta de dados foi realizada entre março e junho de 2018. Os pesquisadores contaram com a concordância do professor em cada sala de aula e realizaram uma breve apresentação da pesquisa aos estudantes. Foi garantido ao participante retirar seu consentimento em qualquer etapa da pesquisa se assim desejasse, lembrando-o da confidencialidade das informações e da manutenção do sigilo. Após essa etapa, foi fornecido o Termo de Consentimento Livre e Esclarecido para assinatura.

Na coleta de dados foi usado um questionário autoaplicável, confeccionado pelos autores com base em questões oriundas dos instrumentos de Oliveira ${ }^{16}$, Frizzo et al. ${ }^{17}$, Pinheiro ${ }^{18}$, Nakazawa et al. ${ }^{19}$ e Gasperin ${ }^{20}$, perfazendo 39 questões, agrupadas em 12 questões sociodemográficas e 27 questões objetivas sobre cuidados paliativos.

Os dados coletados foram inseridos no programa Epi Info, versão 3.5.4 e posteriormente exportados para o software SPSS 20.0 para a análise de dados. As variáveis quantitativas foram descritas por meio de medidas de tendência central e dispersão dos dados. As variáveis qualitativas foram descritas por meio de frequência absoluta e percentual. As diferenças nas proporções foram calculadas por meio do teste de qui-quadrado $\left(X^{2}\right)$, exato de Fisher ou razão de verossimilhança, e as diferenças de médias pelo teste Anova e teste de Tukey para comparações múltiplas, conforme adequação dos dados. O nível de significância estatística adotado foi de 5\% (valor de $\mathrm{p}<0,05)$.

Este estudo foi aprovado pelo Comitê de Ética em Pesquisa com Seres Humanos (CEP) da Unisul, sob o Protocolo $\mathrm{n}^{\circ}$ 2.538.702, em 12 de março de 2018 . 


\section{RESULTADOS}

De um universo de 190 matriculados no internato médico, participaram do estudo 188 (98\%) acadêmicos. A média de idade dos participantes foi de 24,97 (DP $\pm 3,51$ ), variando de 21 a 50 anos de idade. As características sociodemográficas dos participantes do estudo estão apresentadas na Tabela 1.

\begin{tabular}{|c|c|}
\hline $\begin{array}{r}\text { Característica } \\
\text { acadêmicos }\end{array}$ & \\
\hline Dados sociodemográficos & n (\%) \\
\hline Sexo & \\
\hline Masculino & $81(43,1)$ \\
\hline Feminino & $107(56,9)$ \\
\hline Faixa etária & \\
\hline $21-25$ anos & $134(71,3)$ \\
\hline $26-30$ anos & $42(22,4)$ \\
\hline $31-35$ anos & $8(4,2)$ \\
\hline $36-40$ anos & $3(1,6)$ \\
\hline$>40$ anos & $1(0,5)$ \\
\hline Semestre que está cursando & \\
\hline 9o & $49(26,1)$ \\
\hline $10^{\circ}$ & $51(27,1)$ \\
\hline $11^{\circ}$ & $43(22,9)$ \\
\hline $12^{\mathrm{o}}$ & $45(23,9)$ \\
\hline Possui religião/crença & \\
\hline Não & $41(21,8)$ \\
\hline Sim & $147(78,2)$ \\
\hline Se sim, qual religião & \\
\hline Católico & $98(66,7)$ \\
\hline Espírita & $15(10,2)$ \\
\hline Evangélico & $6(4,1)$ \\
\hline Outro & $28(19)$ \\
\hline
\end{tabular}

Dados da pesquisa.

Quanto à percepção dos acadêmicos sobre o processo de morte, $86,2 \%$ afirmaram já ter vivenciado em familiares ou em pessoas com quem mantinham vínculo afetivo. Quando questionados a respeito da decisão de escolha do local adequado para o falecimento, $72,9 \%$ dos participantes afirmaram que a decisão deveria caber ao paciente, juntamente com sua família e seu médico, conforme demonstrado na Tabela 2.

Quando interrogados a respeito de quem deveria decidir sobre o tratamento de um paciente oncológico terminal, no exercício da profissão médica, 70,2\% dos entrevistados optaram por partilhar a decisão entre paciente, familiar e médico, conforme a Tabela 2. De acordo com os semestres em estudo, 69,4\% dos acadêmicos do nono semestre, 70,6\% dos acadêmicos do décimo semestre, $67,4 \%$ dos acadêmicos do décimo primeiro semestre e 73,3\% dos acadêmicos do décimo segundo semestre optaram por essa associação, porém não houve significância estatística.

\section{TABELA 2}

Percepção sobre o processo de morte e decisões no final da vida em acadêmicos do CM-Unisul, 2018

Percepção sobre a morte e decisões no final da vida $\quad$ n (\%)

Já vivenciou o processo de morte em familiares ou pessoas próximas?

$\begin{array}{lc}\text { Sim } & 162(86,2) \\ \text { Não } & 26(13,8)\end{array}$

Você considera importante um paciente oncológico em estado terminal morrer em casa junto com a sua família?

Sim, no lugar em que sempre viveu

Não, pois estaria sem assistência médica

Acredito que o paciente deve decidir juntamente com a família e seu médico

Como médico de um paciente oncológico terminal, quem você gostaria que escolhesse a forma de tratamento desse paciente?

$\begin{array}{lc}\text { Médico } & 2(1,1) \\ \text { Paciente } & 11(5,9) \\ \text { Familiar } & 0(0,0) \\ \text { Paciente e familiar } & 4(2,1) \\ \text { Paciente e médico } & 39(20,7) \\ \text { Paciente, familiar e médico } & 132(70,2)\end{array}$

Caso você fosse o paciente oncológico terminal, quem você gostaria que escolhesse a sua forma de tratamento?

$\begin{array}{lc}\text { Médico } & 6(3,2) \\ \text { Paciente } & 16(8,5) \\ \text { Familiar } & 0(0,0) \\ \text { Paciente e familiar } & 1(0,5) \\ \text { Paciente e médico } & 58(30,9) \\ \text { Paciente, familiar e médico } & 107(56,9)\end{array}$

Dados da pesquisa.

Quando questionados, na posição de pacientes oncológicos terminais, sobre quem gostariam que escolhesse a forma de tratamento, 3,2\% (6) dos entrevistados responderam que prefeririam que a decisão fosse tomada exclusivamente pelo médico (Tabela 2); dentre estes, 83,3\% (5) eram do sexo masculino e $16,7 \%$ (1) eram do sexo feminino (valor de $\mathrm{p}=0,04$ ). Deram a mesma resposta em relação à escolha do tratamento do paciente oncológico terminal, estando na posição de paciente ou de profissional médico, $82 \%$ dos entrevistados.

Entre os entrevistados, 50,5\% relataram despreparo para lidar com o processo de morte de um paciente e depois com a fase de luto dos familiares (Tabela 3), sendo que 10 (5,3\%) associam a morte com derrota, perda e frustração. Verificou-se que $85(45,2 \%)$ entrevistados acreditaram estar despreparados para lidar com o processo de morte e luto, porém não associaram a morte a sentimentos de derrota, perda e frustração. Deste percentual, observou-se que $59(69,4 \%$ ) eram do sexo feminino (valor de $\mathrm{p}=0,005)$. Constatou-se que $93(49,5 \%)$ participantes do 
estudo declararam estar preparados para enfrentar o processo de morte e luto, e acreditar que a morte é um processo natural; destes, $51(54,8 \%)$ eram do sexo masculino (valor de $\mathrm{p}=0,005)$.

\begin{tabular}{|c|c|}
\hline \multicolumn{2}{|c|}{$\begin{array}{l}\text { Vivências do processo de morte e luto pelos acadêmicos } \\
\text { do internato médico do CM-Unisul, } 2018\end{array}$} \\
\hline Vivências sobre morte e luto & n $(\%)$ \\
\hline \multicolumn{2}{|l|}{$\begin{array}{l}\text { Já vivenciou o processo de morte em familiares ou } \\
\text { pessoas próximas? }\end{array}$} \\
\hline Sim & $162(86,2)$ \\
\hline Não & $26(13,8)$ \\
\hline \multicolumn{2}{|l|}{$\begin{array}{l}\text { Você se considera preparado para lidar com a morte de } \\
\text { um paciente e posteriormente com a fase de luto dos } \\
\text { familiares? }\end{array}$} \\
\hline $\begin{array}{l}\text { Sim. Encaro a morte como um processo natural } \\
\text { da vida }\end{array}$ & $93(49,5)$ \\
\hline $\begin{array}{l}\text { Não. Associo a morte com derrota, perda e } \\
\text { frustração }\end{array}$ & $10(5,3)$ \\
\hline $\begin{array}{l}\text { Não, mas não associo a morte com derrota, perda } \\
\text { e frustração }\end{array}$ & $85(45,2)$ \\
\hline
\end{tabular}

Dados da pesquisa.

Quando interrogados sobre o significado de CP, 150 (79,8\%) acadêmicos afirmaram ter conhecimento do assunto, enquanto 38 (20,2\%) afirmaram já ter ouvido falar desta expressão, porém consideraram não ter conhecimento sobre o tema. $\mathrm{O}$ conhecimento sobre o significado de CP de acordo com os semestres em estudo está evidenciado no Gráfico 1, porém não se observou significância estatística na variação entre os semestres em estudo.

Negaram ter recebido, durante a graduação, informações necessárias para controlar sintomas comuns (como dispneia, vômitos e obstipação) nos pacientes em CP 124 (66\%) participantes. Quando questionados sobre ter adquirido, na graduação, informações necessárias para realizar o cuidado de pacientes em situação terminal, 158 (84\%) acadêmicos responderam que não adquiriram conhecimento a respeito do assunto, conforme demonstrado no Gráfico 2.

Quando questionados sobre ter aprendido, na graduação, habilidades de comunicação e atitude médica para informar más notícias aos pacientes e a seus familiares, 151 (80,3\%) acadêmicos responderam que não adquiriram tais competências. Entre estes, 95 (62,9\%) eram do sexo feminino, e $56(37,1 \%)$ eram do sexo masculino (valor de $\mathrm{p}<0,001$ ).

Com o intuito de mensurar o conhecimento do acadêmico acerca da utilização de escalas para mensuração da dor oncológica, da farmacologia do tratamento da dor e dos efeitos adversos das drogas utilizadas no contexto de $\mathrm{CP}$, foram aplicadas 11 questões sobre o tema. Em cada uma delas, considerou-se somente uma opção como correta. A média total de acertos foi de 8,69 (DP $\pm 1,64)$, variando de 3 a 11 .
GrÁFICO 1

Relação do nível de conhecimento de cuidados paliativos com os semestres do internato do CM-Unisul, 2018

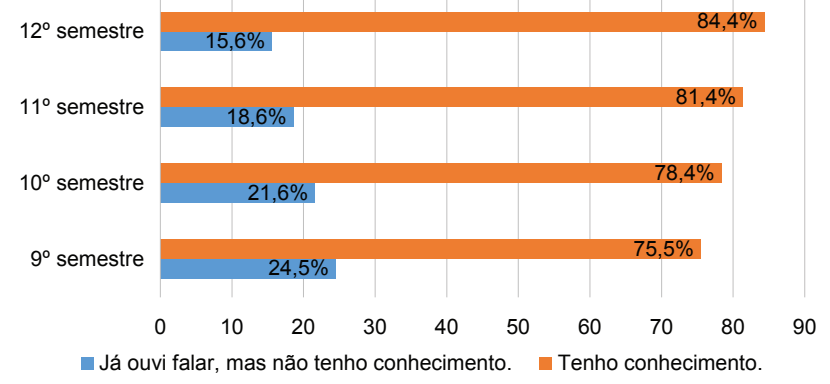

Dados da pesquisa

GrÁfico 2

Conhecimento dos acadêmicos do CM-Unisul, sobre o cuidado de pacientes em situação terminal, 2018

Acredita ter recebido informação suficiente durante a graduação?

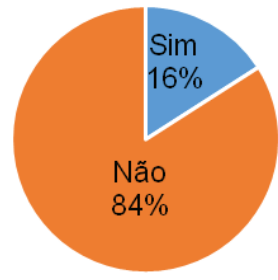

Dados da pesquisa

Observou-se diferença entre as médias de acertos de acordo com os semestres em estudo, conforme demonstrado na Tabela 4. O décimo semestre obteve a maior média de acertos. Na comparação entre os semestres, foram observadas disparidades entre o décimo e o nono semestre $(p=0,04)$, e entre o décimo e o décimo segundo semestre $(p=0,045)$, sendo ambas estatisticamente significativas.

\begin{tabular}{|c|c|c|}
\hline \multicolumn{3}{|c|}{$\begin{array}{l}\text { paliativos de acordo com os semestres } \\
\text { do internato do CM-Unisul, } 2018\end{array}$} \\
\hline Semestre & Média de acertos & Desvio padrão \\
\hline $9^{\circ}$ & 8,24 & 1,931 \\
\hline $10^{\circ}$ & 9,35 & 1,214 \\
\hline $11^{\mathrm{o}}$ & 8,65 & 1,717 \\
\hline $12^{\mathrm{o}}$ & 8,49 & 1,487 \\
\hline
\end{tabular}

Valor de $p=0,005$

Dados da pesquisa. 
Dos 188 entrevistados, 66,5\% afirmaram ter recebido, durante a graduação, informação suficiente para realizar o manejo dos pacientes com dor. Afirmaram ter tido contato com a escala da dor da OMS para mensurar o nível de dor dos pacientes 184 (97,9\%) entrevistados. Entre os que responderam afirmativamente, 173 (94\%) utilizariam a escala sempre ou frequentemente e $134(72,8 \%)$ consideraram a escala uma ferramenta de fácil aplicação. Quanto à escada analgésica da OMS para realizar o manejo de tratamento da dor, $89,9 \%$ dos entrevistados afirmaram ter conhecimento sobre o assunto.

Os acadêmicos que afirmaram conhecer a escada analgésica da OMS responderam a um caso clínico para avaliar a aplicação desse conhecimento, resultando em $82,2 \%$ de respostas corretas, conforme demonstrado na Tabela 5.

\section{TABELA 5}

Conhecimento e aplicação em caso clínico da

escala da dor e da escada analgésica da OMS por acadêmicos do internato do CM-Unisul, 2018

Questão

Você conhece a escada analgésica da OMS para o

manejo da dor?

Não

$19(10,1)$

Sim

$169(89,9)$

Caso tenha respondido sim na questão anterior:

Paciente apresentando dor oncológica leve segundo a

escada da OMS para dor oncológica, sem tratamento

prévio. Qual seria a melhor opção de tratamento de

acordo com a escada analgésica da OMS?

Opioides fracos (codeína/tramadol) + drogas adjuvantes

Analgésicos não opiáceos (aines/analgésicos

simples) + drogas adjuvantes

Opioides fortes (morfina, metadona, fentanil) + drogas adjuvantes

Não soube opinar

Aines: anti-inflamatórios não esteróides.

Dados da pesquisa.

Ao serem interrogados quanto ao conhecimento sobre a escala de performance status (índice de desempenho físico), $22,9 \%$ dos acadêmicos relataram nunca ter ouvido falar sobre o assunto. Observou-se que 54,3\% dos entrevistados responderam já ter ouvido falar sobre o assunto, porém consideraram não ter conhecimento científico sobre o tema. Relataram ter conhecimento sobre essa escala $22,9 \%$ dos participantes.

Consideraram importante a incorporação de conteúdos sobre CP no currículo de Medicina 181 (96,3\%) participantes do estudo. Dos acadêmicos que afirmaram ter conhecimento a respeito do significado de $\mathrm{CP}, 96 \%$ consideraram a incorporação importante. Quando interrogados se gostariam de atuar numa equipe de $C P, 68,1 \%$ responderam que não tinham interesse.

\section{DISCUSSÃO}

Encontrou-se no presente estudo um predomínio do sexo feminino, corroborando estudos realizados em escolas médicas brasileiras, nas quais há um processo de aumento marcante do sexo feminino. Também se observa a inserção das mulheres em carreiras antes predominantemente masculinas ${ }^{21,22}$.

Colocando-se o acadêmico na posição de um paciente oncológico terminal, observou-se predomínio do sexo masculino na decisão de escolha do tratamento tomada somente pelo médico. Esse comportamento corrobora um estudo realizado com pacientes oncológicos na cidade de São Paulo, no qual o gênero pareceu ser um fator importante na capacidade de enfrentamento da doença. Gianini ${ }^{23}$ apresenta que o enfrentamento do estresse varia em cada situação de forma individual, porém as mulheres tendem a usar estilos de enfrentamento mais focados na emoção, com preferência pelo apoio social, enquanto os homens são socialmente cobrados para assumir os problemas de forma mais ativa e orientada para a restauração ou cura.

Observou-se associação entre o sexo masculino e o preparo para lidar com o processo de morte e luto e as habilidades para comunicar notícias desfavoráveis; já no sexo feminino, se evidenciou associação com o despreparo para lidar com o mesmo processo. Há uma psicopatologia - conhecida como Síndrome do Impostor - na qual a pessoa desconfia das próprias competências e se considera aquém das capacidades, não conseguindo aceitar suas conquistas como mérito próprio. Esse fenômeno não é exclusivo do sexo feminino, porém parece ser prevalente e intenso em mulheres de alto desempenho, sendo frequente na área acadêmica e podendo resultar em ansiedade e falta de autoconfiança, comprometendo a performance do indivíduo ${ }^{24,25}$. Um estudo que avaliou a disparidade de gênero referente ao reconhecimento de residentes por suas publicações evidenciou que a minoria era destinada a profissionais do gênero feminino e que as mulheres eram menos propensas a defender suas realizações e também possuíam percepção inferior sobre sua qualidade de trabalho em comparação aos homens ${ }^{26}$.

Em relação a outras variáveis sociodemográficas, houve predomínio da religião católica. A literatura mostra que a religiosidade e a espiritualidade do profissional de saúde colaboram para a valorização da dimensão espiritual do paciente ${ }^{27}$. Entretanto, não houve associação com significância estatística entre ter religião/crença e preparo para lidar com a morte e luto, corroborando um estudo realizado no curso de Medicina da Universidade Federal do Rio Grande do Sul ${ }^{28}$. A maioria dos participantes afirmou já ter vivenciado o processo de morte em pessoas com quem mantinha vínculo afetivo. No 
presente estudo, porém, não foi realizada a investigação sobre a causa da morte, sendo que o convívio com pessoas que necessitam de CP é capaz de, possivelmente, despertar interesse pela área e influenciar o desempenho dos estudantes.

Estando no papel de médico de um paciente oncológico terminal, a maioria dos entrevistados preferiu que a decisão da escolha de tratamento desse paciente fosse tomada pelo conjunto paciente, familiar e médico. Respostas semelhantes foram obtidas no presente estudo quando questionado se o paciente fosse o próprio acadêmico. Houve predomínio de entrevistados que mantiveram essa resposta se estivessem na posição de paciente ou de médico, demonstrando uma possível aptidão de identificação e empatia com os pacientes.

Outra pesquisa, que abrangeu todas as fases do curso de Medicina, demonstra prevalência da escolha do tratamento também por paciente, médico e familiar, na posição tanto de médico $(72,9 \%)$, como de paciente $(66,2 \%)^{17}$. Ainda, em estudo com acadêmicos do décimo primeiro e do décimo segundo semestre de Medicina, a maioria dos estudantes optou por escolha semelhante ${ }^{29}$. Nesse contexto, a maioria dos participantes do CM-Unisul respondeu que o local de morte deve ser decidido pelo paciente juntamente com sua família e seu médico, corroborando pesquisas nacionais ${ }^{17,29,30}$. Tal resultado demonstra que os acadêmicos de Medicina reconhecem a importância da participação do familiar no auxílio aos pacientes. A família compõe um dos alicerces da assistência prestada aos pacientes em CP e deve ser integrada à equipe ${ }^{31}$.

Mais da metade dos participantes relataram estar despreparados para lidar com o processo de morte de um paciente e posteriormente com a fase de luto dos familiares, assim como encontrado na Universidade Uninove, na qual 76\% dos entrevistados se consideraram despreparados para essa situação ${ }^{30}$. Ademais, em estudo da Universidade Severino Sombra, 42,7\% dos estudantes se consideraram parcialmente preparados para enfrentar essas circunstâncias ${ }^{29}$. Esse despreparo frente ao processo de morte e luto possivelmente se deve ao ensino focado no tratamento curativo, bem como à carência de discussões sobre a temática durante a graduação médica.

Maior parcela dos acadêmicos negou ter adquirido informações suficientes para o cuidado de pacientes terminais, de modo semelhante a um estudo com 50 acadêmicos do quinto e sexto anos de um curso de Medicina no Estado de São Paulo, no qual $83 \%$ negaram ter recebido tais informações durante a graduação. Portanto, presumivelmente, a abordagem desse conteúdo se encontra aquém do ideal, necessitando maior atenção ${ }^{18}$.

Os acadêmicos do CM-Unisul obtiveram um nível adequado de conhecimento a respeito da farmacologia relaciona- da ao tratamento da dor, avaliado por meio de 11 questões objetivas, diferentemente de um estudo com acadêmicos do primeiro, quarto e sexto anos, em Brasília, realizado por meio da aplicação de um questionário com 19 questões, no qual o nível de conhecimento foi considerado insatisfatório, com menor porcentagem de acertos ${ }^{32}$. Tal discrepância pode se dever à diferença de instrumento de coleta de dados, à população estudada ou, ainda, à forma como o assunto é abordado nas instituições.

Houve diferença da média de acertos entre os semestres participantes, sendo que o décimo semestre apresentou maior média. No nono semestre do curso avaliado, há, durante o estágio de Clínica Médica e Clínica Cirúrgica, um período em que os acadêmicos permanecem em contato direto com pacientes internados em âmbito hospitalar no setor de Oncologia e revisam assuntos voltados à temática de CP. Durante a coleta de dados, somente uma pequena parcela dos acadêmicos do nono semestre havia participado dessa atividade, enquanto os acadêmicos do décimo semestre haviam participado em sua totalidade, o que, possivelmente, influenciou o desempenho dos entrevistados. Esse resultado vai ao encontro de um estudo da Universidade São Francisco, em São Paulo, no qual participaram 240 acadêmicos e que constatou que as habilidades em comunicação e multidisciplinaridade em CP foram mais bem desempenhadas pelo grupo que recebeu treinamento completo, teórico e prático ${ }^{33}$. Possivelmente, os acadêmicos do décimo segundo semestre do CM-Unisul tiveram resultado inferior aos alunos do décimo por terem passado pelas disciplinas que abordaram essa temática havia mais tempo, o que pode ter ocasionado o esquecimento de determinados conceitos.

$\mathrm{O}$ conhecimento relativo à expressão " $\mathrm{CP}$ " evoluiu ao longo dos semestres do internato médico, assim como na Universidade do Oeste de Santa Catarina, podendo-se inferir que o acadêmico aprimora o conhecimento ao longo do internato médico e do contato com os pacientes, porém no presente estudo não houve significância estatística ${ }^{17}$.

Quando questionados sobre assuntos considerados "verdades ou mitos" em CP, os acadêmicos tiveram bom desempenho. Nakazawa e colaboradores ${ }^{19}$ desenvolveram um teste cuja validade foi comprovada numa amostra de 773 participantes e com questões similares às avaliadas no presente estudo. No estudo japonês, essas questões tiveram um percentual inferior de acertos, com até $73 \%$ de respostas corretas. Nesse estudo, os participantes eram profissionais atuantes na enfermagem, podendo os resultados diferir devido à população e ao local, demonstrando bom domínio sobre essa temática nos acadêmicos do internato médico do CM-Unisul. 
O controle da dor é prioridade no tratamento do paciente em CP em virtude de seu impacto positivo sobre a qualidade de vida e da contribuição para que o paciente tolere melhor o tratamento oncológico ${ }^{34}$. O manejo inadequado da dor pode ser atribuído a barreiras relacionadas aos profissionais de saúde, aos pacientes e ao sistema de saúde. As barreiras comuns relacionadas ao profissional incluem baixa avaliação da dor, falta de conhecimento e habilidade, e relutância dos médicos em prescrever opioides ${ }^{35}$.

A OMS recomenda critérios básicos para a prescrição de analgésicos, um modelo da década de 1990 que é eficaz até os dias atuais: "pela boca, pelo relógio, pela escada, individualização e atenção ao detalhe" ${ }^{\prime \prime 36}$. Na prática de $\mathrm{CP}$, sempre que possível, deve-se preferir o tratamento mais simples e menos invasivo; portanto, de modo geral, medicações orais são preferíveis $^{34}$. No presente estudo, $41 \%$ dos participantes sabiam que a via preferencial para administração de morfina é a via oral, enquanto $39 \%$ acreditavam que a via endovenosa seria a preferencial, possivelmente por associarem o cuidado administrado em âmbito hospitalar com o uso de medicações por via intravenosa.

A maioria dos participantes tinha conhecimento sobre a farmacologia do uso de opioides, corroborando dados encontrados em um estudo com 160 acadêmicos do internato médico do CM-Unisul em 2005 ${ }^{20}$. Observa-se que o conhecimento dos acadêmicos do internato médico do CM-Unisul no ano de 2018 se manteve semelhante ao encontrado há 13 anos, demonstrando a permanência de uma boa instrução referente a esta temática na instituição de ensino.

A maioria dos acadêmicos considerou a escala da dor uma ferramenta de fácil aplicação, e houve boa aplicabilidade do conhecimento em caso clínico. Esse achado sugere que os acadêmicos detêm bom conhecimento sobre o manejo do paciente com dor e que utilizarão essa abordagem em seu futuro profissional, exercendo, assim, melhor controle do sintoma doloroso nos pacientes.

A minoria dos acadêmicos afirmou ter conhecimento sobre a escala de performance status (índice de desempenho físico), corroborando um estudo realizado na Universidade do Oeste de Santa Catarina no qual somente 15,8\% dos acadêmicos afirmaram conhecê-la, apontando a necessidade de aprimorar essa temática na graduação médica ${ }^{17}$. Porém, uma abordagem mais abrangente de $\mathrm{CP}$ exigiria treinamento específico dos docentes e, possivelmente, um cronograma de aulas diferenciado.

Os acadêmicos, em sua maioria, consideraram importante a incorporação de conteúdos sobre CP no currículo de Medicina, mas afirmaram não ter interesse em atuar numa equipe de CP. Esse resultado, presumivelmente, aponta que os acadêmicos reconhecem a relevância desse aprendizado para o exercício de suas carreiras profissionais e, portanto, consideram importante estar preparados, independentemente da área de atuação desejada. Porém, não há preferência pela atuação na área, provavelmente pela complexidade de lidar com pacientes crônicos e seus familiares, o que exige uma abordagem humanizada e traz reflexões e questionamentos sobre a vida, e, por conseguinte, a morte.

É válido lembrar que o presente estudo apresenta limitações, como o delineamento transversal, que não possibilita o acompanhamento e a evolução do conhecimento de cada acadêmico de forma direta. Também, quando os participantes foram interrogados sobre terem vivenciado o processo de morte em familiares ou pessoas próximas, não se levou em conta a causa da morte, fato que pode interferir no conhecimento ou interesse por $\mathrm{CP}$.

\section{CONCLUSÃO}

O ensino sobre a temática de $\mathrm{CP}$ durante a graduação do CM-Unisul resultou em um conhecimento adequado dos acadêmicos do internato. Entretanto, observam-se dificuldades frente ao processo de morte e insegurança na abordagem de comunicações e atitude médica. É necessário aprimorar o ensino de competências e habilidades na área, enfatizando o cuidado universal centrado no paciente e não somente na cura de doenças, bem como mobilizar esforços a fim de incentivar a autoestima dos acadêmicos.

\section{REFERÊNCIAS}

1. World Health Organization (WHO). Active Ageing: A Policy Framework. Aging Male. [Internet]. 2002;5(1):137. Disponível em: http://apps.who.int/iris/bitstream/10665/67215/1/WHO_NMH_NPH_02.8.pdf Acesso em 20 Out 2017

2. Prince MJ, Wu F, Guo Y, Robledo LMG, Q’Donnell M, Sullivan $\mathrm{R}$, et al. The burden of disease in older people and implications for health policy and practice. Lancet. 2015;385(9967):549-62.

3. Ministério da Saúde (BR). Secretaria de Vigilância em Saúde. Departamento de Análise de Situação de Saúde. Plano de ações estratégicas para o enfrentamento das Doenças Crônicas Não Transmissíveis (DCNT) no Brasil 20112022. Brasília: Ministério da Saúde, 2011.

4. Galriça Neto I. Princípios e filosofia dos Cuidados Paliativos. In: Barbosa A, Galriça Neto I, organizadores. Manual de Cuidados Paliativos. 2. ed. Lisboa: Faculdade de Medicina da Universidade de Lisboa, 2010. p.1-42. 
5. Matsumoto DY. Cuidados paliativos: conceitos, fundamentos e princípios. In: Carvalho RT, Parsons HA, organizadores. Manual de cuidados paliativos da ANCP. Rio de Janeiro: Diagraphic; 2012. p. 23-30.

6. Academia Nacional de Cuidados Paliativos. Cuidados Paliativos: conceito, fundamentos e princípios. In: Manual de cuidados paliativos. Rio de Janeiro: Diagraphic; [manual na Internet]. 2009;14-19 Disponível em: http://biblioteca. cofen.gov.br/wp-content/uploads /2017/05/Manual-de-cuidados-paliativos-ANCP.pdf Acesso em 28 Out 2017

7. Du Boulay S. Changingthe face of death. The story of Cicely Saunders. 2nd. ed. Great Britain: Brightsea Press. 2007. $24 \mathrm{p}$.

8. World Health Organization (WHO). Connor SR, SepulvedaBermedo MC. Global atlas of palliative care at the end of life. [Internet]. 2014. Disponível em: http:/ / www.who. int/nmh/Global_Atlas_of_Palliative_Care.pdf Acesso em 28 Out 2017

9. National Consensus Project for Quality Palliative Care. Clinical Practice Guidelines for Quality Palliative Care. 3rd ed. Pittsburgh, PA: National Consensus Project for Quality Palliative Care; [Internet]. 2013. Disponível em: https://www.hpna.org/multimedia/NCP_Clinical_Practice_Guidelines_3rd_Edition.pdf Acesso em 28 Out 2017

10. Fonseca A, Geovanini F. Cuidados paliativos na formação do profissional da área de saúde. Rev. bras. educ. med. 201337 (1): 120-125.

11. Conselho Federal de Medicina (CFM). Código de ética médica: resolução CFM nº 1.931, de 17 de setembro de 2009. Conselho Federal de Medicina. Brasília: Conselho Federal de Medicina; 2010. 70p.

12. The Economist Intelligence Unit. The 2015 Quality of Death Index Ranking palliative care across the world. Econ [Internet]. 2015 Disponível em: http://www.apcp.com. pt/uploads / 2015-EIU-Quality-of-Death-Index-Oct-6-FINAL.pdf Acesso em 20 Out 2017

13. Mello AAM, Silva LC. A estranheza do médico frente à morte: lidando com a angústia da condição humana. Rev. abordagem Gestalt. 2012;18(1):52-60.

14. Duarte AC, Almeida DV, Popim RC. A morte no cotidiano da graduação: um olhar do aluno de medicina. Interface (Botucatu). 2015;19(55):1207-1219.

15. Horowitz R, Gramling R, Quill T. Palliative care education in US medical schools. Medical Education. 2014;48(1):5966.

16. Oliveira DS. A percepção do estudante de medicina sobre a morte e o morrer [monografia]. Salvador (BA). Universidade Federal da Bahia, Curso de Medicina; 2017.
17. Frizzo K, Bertolini G, Caron R, Steffani JA, Bonamigo EL. Percepção dos acadêmicos de medicina sobre cuidados paliativos de pacientes oncológicos terminais. RevBioethikos. 2013;7(4):367-375

18. Pinheiro TRSP. Avaliação do grau de conhecimento sobre cuidados paliativos e dor dos estudantes de medicina do quinto e sexto anos. O Mundo da Saúde. 2010;34(3):320-326.

19. Nakazawa Y, Miyashita M, Morita T, Umeda M, Oyagi Y, Ogasawara T. The palliative care knowledge test: Reliability and validity of na instrument to measure palliative care knowledge among health professionals. Palliative Medicine. 2009; 23(8): 754-766.

20. Gasperin AAM. Avaliação do conhecimento que os acadêmicos do internato médico do curso de medicina da Unisul têm sobre o tratamento da dor oncológica, no ano de 2005. Tubarão (SC). Monografia [Graduação em Medicina] - Universidade do Sul de Santa Catarina; 2005.

21. Fiorotti KP, Rossoni RR, Miranda AE. Perfil do estudante de Medicina da Universidade Federal do Espírito Santo, 2007. Revbraseduc med. 2010;34(3):355-362.

22. Ricoldi A, Artes A. Mulheres no ensino superior brasileiro: espaço garantido e novos desafios. Ex aequo. 2016;33:149161.

23. Gianini MMS. Câncer e gênero: enfrentamento da doença [dissertação]. São Paulo: Pontifícia Universidade Católica de São Paulo, 2006.

24. Clance PR, Imes S. The Imposter Phenomenon in High Achieving Women: Dynamics and Therapeutic Intervention Psychotherapy Theory, Research and Practice. 1978;15(3):1-8.

25. Kauati A. Síndrome do impostor e a vida acadêmica. Interparadigmas. 2013;1(1): 75-88.

26. Rotenstein LS, Berman RA, Katz JT, Yialamas MA. Making the Voices ofFemale Trainees Heard. Ann Intern Med. 2018;169(5):339-340.

27. Longuiniere ACFDL, Yarid SD, Silva ECS. Influência da religiosidade/espiritualidade dos profissionais da saúde na valorização da dimensão espiritual do paciente crítico. Revenferm UFPE. 2017;11(6):2510-2517

28. Azeredo NSG; O acadêmico de medicina frente a morte: questões para se (re)pensar a formação [dissertação]. Porto Alegre: Universidade Federal do Rio Grande do sul. Faculdade de Medicina. Programa de Pós Graduação em Ciências Médicas: Pediatria, 2007.

29. Alves Jr VD, Gutterres DB, Corrêa LGP, Ajovedi GB, de Souza MCA. Cuidados Paliativos em pacientes terminais: Conhecimentos de estudantes de medicina. Cuidados Paliativos 2018;5(1):68-75 
30. Moraes SAF, Kairalla MC. Avaliação dos conhecimentos dos acadêmicos do curso de Medicina sobre os cuidados paliativos em pacientes terminais. Einstein. 2010;8(2):162-7.

31. Matos JC, Borges MS. A família como integrante da assistência em cuidado paliativo. Revenferm UFPE. 2018;12(9):2399-406.

32. Lemos CFP, Barros GS, Melo NCV, Amorim FF, Santana ANC. Avaliação do Conhecimento em Cuidados Paliativos em Estudantes durante o Curso de Medicina.Revbraseduc med. 2017;41 (2): 278-282.

33. Malta R, Rodrigues B, Priolli DG. Paradigma na Formação Médica: Atitudes e Conhecimentos de Acadêmicos sobre Morte e Cuidados Paliativos.Revbraseduc med. 2018;42(2):34-44.

34. Wiermann EG, Diz MPE, Caponero R, Lages PSM, de Araujo CZS, Bettega RTC, et al. Consenso Brasileiro sobre o manejo da dor relacionada ao câncer. RevBrasOncolClin. 2014;10(38):133-43.

35. Know JH. Overcoming Barriers in CancerPain Management. Journal of Clinical Oncology 2014;32(16):1727-1733

36. World Health Organization (WHO) Expert Committee on Cancer Pain Relief and Active Supportive Care. Cancer pain relief and palliative care: report of a WHO expert committee [meeting held in Genevafrom 3 to 10 July 1989], 1990. Disponível em: http://www.who.int/iris/handle/10665/39524 Acesso em 20 Out 2018

\section{CONTRIBUIÇÃO DOS AUTORES}

Larissa Chaiane Orth: elaboração/edição, coleta de dados. Eder Yukio Haragushiku: elaboração/edição, coleta de dados. Inês Carolina Siqueira Freitas: elaboração/edição, coleta de dados. Micheli Cristiane Hintz: elaboração/edição, coleta de dados. Chaiana Esmeraldino Mendes Marcon: elaboração/ edição, orientação da pesquisa. José Francisco Teixeira: elaboração/edição, orientação da pesquisa.

\section{CONFLITO DE INTERESSES}

Os autores declaram não haver conflito de interesses.

\section{ENDEREÇO PARA CORRESPONDÊNCIA}

Larissa Chaiane Orth

Universidade do Sul de Santa Catarina - Av. José Acácio

Moreira, 787

CEP:88704-900

Dehon, Tubarão-SC

Email: larissaorth1@gmail.com ; larissa.orth@unisul.br 